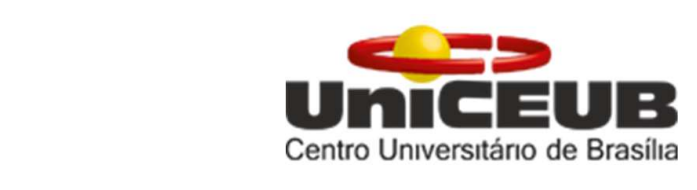

CENTRO UNIVERSITÁRIO DE BRASÍLIA - UniCEUB

PROGRAMA DE INICIAÇÃO CIENTÍFICA

MATEUS MEDEIROS LEITE

INFLUÊNCIA DE POLIMORFISMOS GENÉTICOS NA EXPRESSÃO DE ENOS, APOE E ECA EM RESPOSTA AO TREINAMENTO DE FORÇA EXCÊNTRICO EM IDOSAS OBESAS.

BRASÍLIA

2018 


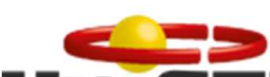

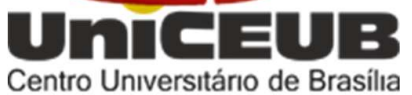

MATEUS MEDEIROS LEITE

INFLUÊNCIA DE POLIMORFISMOS GENÉTICOS NA EXPRESSÃO DE ENOS, APOE E ECA EM RESPOSTA AO TREINAMENTO DE FORÇA EXCÊNTRICO EM IDOSAS OBESAS.

Relatório final de pesquisa de Iniciação Científica apresentado à Assessoria de Pós-Graduação e Pesquisa.

Orientação: Alessandro de Oliveira Silva

BRASÍLIA

2018 


\title{
INFLUÊNCIA DE POLIMORFISMOS GENÉTICOS NA EXPRESSÃO DE ENOS, APOE E ECA EM RESPOSTA AO TREINAMENTO DE FORÇA EXCÊNTRICO EM IDOSAS OBESAS.
}

\author{
Mateus Medeiros Leite - UniCEUB, PIC Institucional, aluno bolsista \\ mateus4100@gmail.com (mateus.leite@sempreceub.com)
}

\author{
Alessandro de Oliveira Silva - UniCEUB, professor orientador \\ silva.alessandro.oliveira@gmail.com (e-mail institucional - @ceub.edu.br)
}

\author{
Silvana SchwerzFunghetto - UnB, professor colaborador \\ silvana.funghetto@gmail.com
}

O aumento da expectativa de vida nos últimos anos associa-se a prevalência da obesidade e outras doenças crônicas não transmissíveis, decorrentes de processos multifatoriais com influência da genética e estilo de vida adotado. O objetivo do presente estudo foi investigar como os genótipos identificados nos polimorfismos de eNOS, APOE e ECA podem influenciar na resposta de CK após TFE. Participaram 86 mulheres idosas obesas $(69,24 \pm 4,94$ anos). Para caracterização foi avaliada a composição corporal com DEXA, Colesterol total, HDL, LDL, VLDL e Triglicérides. As idosas completaram um protocolo de TFE com $110 \%$ de 10 RM apenas na fase excêntrica. Foi avaliada a resposta de CK nos momentos pré-exercício, pós0, pós3hrs e pós24hrs. As voluntárias foram dividas conforme polimorfismo genético de eNOS, APOE e ECA. Para comparação das características dos grupos por genótipos foi utilizado teste t para amostras independentes quando verificada normalidade e teste $U$ de Mann-Whitney quando em anormalidade. ANOVA fatorial de delineamento misto $2 \times 4$ (genótipo $x$ momento) foi empregada para avaliar a resposta de CK nos genótipos de cada polimorfismo nos quatro momentos através do programa SPSS 25.0 adotando como significância $p \leq 0,05$. Para os genótipos GG e TG/TT de eNOs, e3/e3 e e3/e4-e2/e3 de APOE não foram observadas diferenças significativas paras as variáveis de caracterização $(p>0,05)$, já as voluntárias com genótipo DI/II da ECA apresentaram maior idade em relação ao genótipo DD $(p=0,034)$. 0 TFE utilizado induziu a um aumento de CK para o genótipo TG/TT de eNOS no momento Pós 24 horas em relação a Pós0 e Pós3hrs ( $p=0,006)$, de 90,85 \pm 36,65 U/l e 91,75 $\pm 35,58 \mathrm{U} / \mathrm{I}$ respectivamente para 107,74 $\pm 42,35$ U/I Pós24hrs. O genótipo e3/e3 de APOE demonstrou um aumento significativo de 90,81 \pm 35,08 U/I Pós0, 89,58 \pm 35,00 U/I Pós3hrs para 105,22 \pm $43,70 \mathrm{U} / \mathrm{I}$ no momento Pós24hrs ( $p=0,013 ; p=0,002$ ) respectivamente. CK para o genótipo DI/II de ECA aumentou significativamente de 87,88 \pm 33,01 U/I Pré, 88,26 \pm 3 8,18UI Pós0 e $88,64 \pm 35,99$ Pós3hrs em relação ao momento Pós24hrs 104,76 $\pm 46,27 \mathrm{U} / \mathrm{I}(\mathrm{p}=0,042 ; \mathrm{p}=$ $0,011 ; p=0,008)$ respectivamente. O protocolo de TFE parece induzir um aumento de CK a partir de 24 horas após o exercício para os genótipos TG/TT de eNOS, e3/e3 de APOE e DI/II de ECA, sem diferença significativa entre os genótipos dos respectivos polimorfismos.

Palavras-Chave: Envelhecimento. Obesidade. Treinamento de força. Polimorfismos genéticos. 


\section{LISTA DE ABREVIATURAS}

(CK) - Creatina Quinase.

(DEXA) - Absortometria Radiológica de Dupla Energia.

(TFE)- Treinamento de Força Excêntrico.

(10 RM) - Teste de 10 Repetições Máximas.

(U/I) - Unidade por litro.

(IMC) - Índice de Massa Corporal.

(\%G) - Percentual de Gordura.

(MM) - Massa Magra.

(CTotal) - Colesterol Total.

(HDL) - Lipoproteína de Alta Densidade.

(LDL) - Lipoproteína de Baixa Densidade.

(VLDL) - Lipoproteína de Muito Baixa Densidade.

(TGL) - Triglicerídeo.

(FM) - Força máxima

(ON) - Óxido Nítrico

(eNOS) - Sintase Endotelial de Óxido Nítrico

(ECA) - Enzima Conversora de Angiotensina

(APOE) - Apolipoproteína-E

(DCNT) - Doenças Crônicas Não Transmissíveis

(DCV) - Doenças Cardiovasculares

(HAS) - Hipertensão Arterial Sistêmica

(DM) - Diabetes Mellitus

(IL-6) - Interleucina-6 


\section{SUMÁRIO}

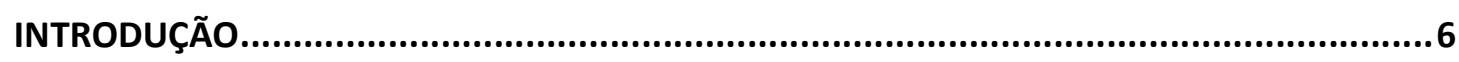

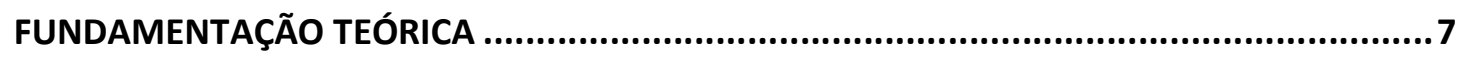

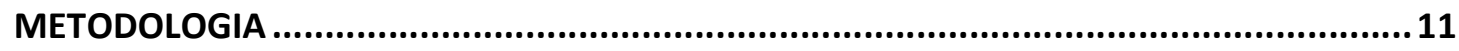

Local de realização da pesquisa .................................................................... 11

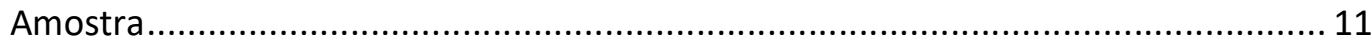

Instrumentos e procedimentos metodológicos ............................................ 12

Avaliação da composição corporal.............................................................. 12

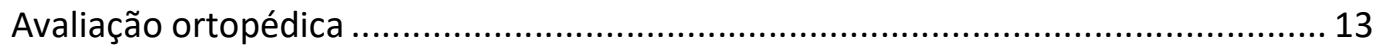

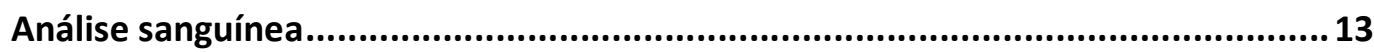

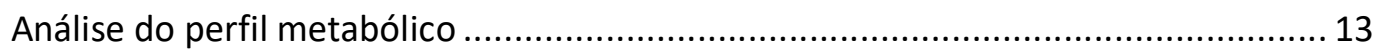

Análise do perfil genotípica ...................................................................... 13

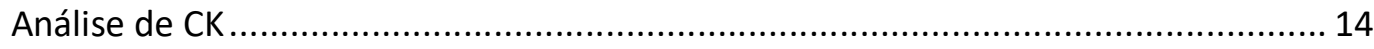

Procedimentos experimentais de Atividade Física .............................................14

Fase de adaptação ao exercício ................................................................... 15

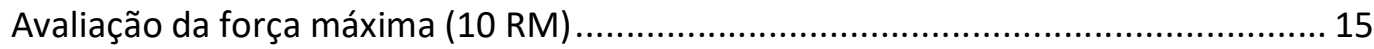

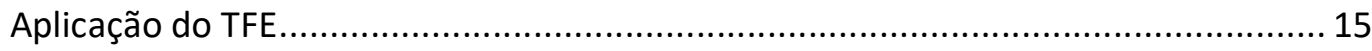

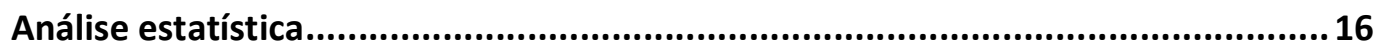

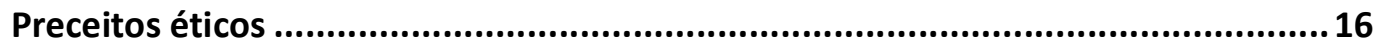

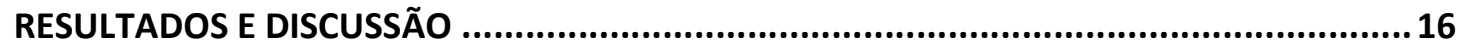

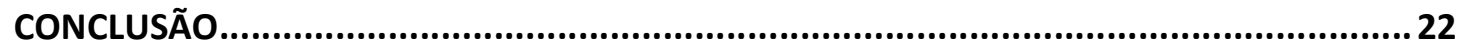

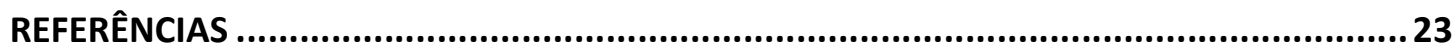




\section{INTRODUÇÃO}

O perfil populacional em escala mundial passa por uma modificação ao longo dos últimos anos, com aumento da expectativa de vida e crescente prevalência da obesidade associada a outras doenças crônicas não transmissíveis (DCNT) e causas de morbimortalidade. Em especial, modificações na composição corporal associadas ao envecheciemento, perfil metabólico e inflamatório parecem ser concomitantes com o desenvolvimento de DCNT, a saber, obesidade, hipertensão arterial sistêmica (HAS), doenças cardiovasculares (DCV), diabetes mellitus (DM), dislipidemias e câncer (CAMARANO, 2007; GRUNDY, 2002; CAVALCANTI, 2010).

O envelhecimento é um processo natural de deterioração do organismo seguido de mudanças físicas substanciais, ocorridas na composição corporal como perda de massa muscular, ganho de peso, além de perdas na capacidade cognitiva, alterações psicológicas e aumento de possibilidades para desenvolvimento de doenças crônicas (DC) (FIORE et al., 2006; MENEZES et al., 2013; MATSUDO, 2001; HAYFLINCK, 1996).

Vários são os fatores influentes para o desenvolvimento de DCNT associadas ao envelhecimento. Como em um ciclo, alterações na capacidade funcional, sistema musculoesquelético, sistema cardiovascular, força e resistência muscular, aspecto físicocognitivo, irão interferir na autonomia e independência para realização das atividades de vida diária, diminuindo o nível de atividade física e consequentemente maior predisposição à DCNT (BALSAMO; SIMÃO, 2007).

Com isso, dentre as opções para prevenção e tratamento de DCNT bem como para a promoção do envelhecimento ativo e saudável, é necessário que haja mudanças nos hábitos diários com a implementação de um programa de exercício físico e uma alimentação balanceada, com resultados positivos na composição corporal, perfil metabólico e melhora na capacidade funcional e saúde mental da população idosa (BAUMAN et al., 2016; RAUEN et al., 2008).

Neste sentido programas de treinamento de força surgem como uma estratégia com uma contribuição substancial para promoção de uma vida saudável e com qualidade para desenvolvimento de atividades da vida diária, com aumento na força, massa muscular e óssea além de diminuição do estado inflamatório sistémico, diminuição de pressão arterial entre 
outros benefícios especialmente em idosos (BALSAMO; SIMÃO, 2007; CHODZKO-ZAJKO et al., 2009; STRASSER; SCHOBERSBERGER, 2011; FUNGHETTO et al., 2013; MOTA et al., 2013).

Diante disto, segundo o Colégio Americano de Medicina do Esporte (ACSM, 2007) dentro da prescrição do treinamento existem variáveis importantes na qual deve-se atentar para uma correta prescrição de acordo com o estado do indivíduo e os objetivos do programa de treinamento. Dentre estas variáveis estão as ações musculares, sejam elas concêntricas, excêntricas ou isométricas, que dão estímulos diferentes ao sistema neuromuscular assim como promovem respostas diferentes ao organismo (PETERSEN; PEDERSEN, 2006; PERDERSEN 2007; FUNGHETTO et al., 2013).

Assim, o exercício excêntrico é postulado como um estímulo que ativa um menor número de fibras musculares e consequentemente, um menor número de unidades motoras é recrutado nesta ação, produzindo maior força com maior aproveitamento do movimento e menor estimulação neural. Assim observa-se uma maior eficiência neuromuscular, além de evidências que demonstram a preservação da força excêntrica em adultos mais velhos (KRAMER et al, 2001; REMAUD; CORNU, 2005; DURAND et al, 2003; PURKAYASTHA et al, 2006, SCHMITZ; WESTWOOD, 2001; ROIG et al., 2010).

Sabendo que a obesidade é decorrente de um processo multifatorial influenciada por fatores genéticos que desempenham um papel importante no desenvolvimento desta associada aos hábitos alimentares e estilo de vida adotados (XIA; GRANT, 2013; BELL; WALLEY; FROGUEL, 2005), e que a produção de creatina quinase (CK) é induzida pelas micro-lesões nos músculos ocasionadas pelo treinamento de força (BAIRD et al., 2012), o objetivo do presente estudo foi investigar como os genótipos identificados nos polimorfismos de eNOS, APOE e ECA podem influenciar na resposta de CK após TFE, além de comparar as características antropométricas e bioquímicas de acordo com os genótipos destes polimorfismos.

\section{FUNDAMENTAÇÃO TEÓRICA}

A obesidade é um fator de risco para o desenvolvimento de diversas outras DCNT, como DM, dislipidemias, alguns tipos de câncer, osteoartrite, problemas respiratórios, alterações endócrinas e DCV (HALPERN; MANCINI, 2002). Além da obesidade, as alterações no sistema cardiovascular, estão relacionadas ao que parece com um aumento na massa e rigidez da parede ventricular esquerda e mudanças na capacidade vascular, como a 
vasodilatação, mediada pela expressão de óxido nítrico (ON), produzido pela sintase endotelial de óxido nítrico (eNOS) com importante função no controle da pressão sanguínea (BALSAMO; SIMÃO, 2007; CHANNON; GUZIK, 2002; RUSH et al., 2005).

O desenvolvimento de DCV, doenças renais, doenças coronarianas, aterosclerose, infarto agudo do miocárdio, HAS e acidente vascular cerebral estão relacionados a fatores genéticos e ambientais, podendo associar-se a expressão de eNOS e óxido nítrico ON e seus polimorfismos, que são diferentes variações em uma sequência de DNA em um determinado local cromossômico. Alterações na expressão de eNOS contribuem para uma disfunção endotelial diminuindo significativamente sua quantidade e/ou atividade enzimática e produção de ON, fator importante na regulação do fluxo sanguíneo, termogênese, modulação da atividade neuronal e um mecanismo essencial para limitar a inflamação (COLOMBO et al., 2002; ZHANG et al., 2006; LEE et al., 2012; ICHIHARA et al., 1998; FOWKES et al., 2000; FARACl; BRIAN, 1994; FÖRSTERMANN et al., 1998; ANDREW; MAYER, 1998).

Além disto, outro fator de grande impacto para o desenvolvimento de DCV e doenças renais pode ser demonstrado em variações genéticas no gene da enzima conversora de angiotensina (ECA), o que pode modificar sua capacidade de metabolizar peptídeos vasoativos e parecem ser fortes candidatos para influenciar fenótipos musculares, potência e velocidade em populações idosas, porém alguns estudos não identificaram a influência do polimorfismo I/D do gene da ECA sobre a força e o volume muscular em mulheres sedentárias de meia idade (SOUBRIER, 2013; PEREIRA et al., 2013; TIBANA et al., 2014).

Esta enzima, é chave no sistema renina-angiotensina, convertendo angiotensina (AN) I em AN II, um vasoconstritor e retentor de sódio, e inativando a bradicinina (um vasodilatador) com efeitos na função musculoesquelética e de grande importância para controle da pressão arterial e tônus vascular. Além disso, a AN II também aumenta a hipertrofia induzida por sobrecarga do músculo esquelético e hipertrofia ventricular esquerda. Seu polimorfismo genético, definido com base na presença [inserção- I] ou ausência [deleção- D], demonstra haver maior atividade de ECA em indivíduos com a presença do alelo D do que naqueles com o alelo I e D/I, estes diretamente relacionados com a HAS (ZEE et al., 1992; GORDON et al., 2001; WESTERKAMP; GORDON, 2005; HERNÁNDEZ et al., 2003; MONTGOMERY et al., 1998; RIGAT et al., 1990).

Ainda associado com desenvolvimento de DCV e doenças coronarianas acarretadas por disfunções endoteliais e inflamações ocasionadas pelo aumento de colesterol sanguíneo 
e acúmulo de lipoproteínas de baixa densidade (LDL) favorecendo o desenvolvimento de placas aterogênicas (PETROVAN et al., 2012), está a ação da Apolipoproteína-E (APOE), envolvida na regulação hemostática, metabolismo e transporte de lipoproteínas e na remoção de LDL no plasma, e os alelos polimórficos, especialmente o alelo e4 em seu gene, está associados com DCV (ASGHAR et al., 2013; COOPER et al. 2008; MARTINELLI, at al., 2009; SMITH, 2000).

Diante do exposto, foi realizada uma revisão sistemática integrativa da literatura com busca de artigos foi realizada em abril de 2017 nas seguintes bases eletrônicas: Pubmed, Ebsco e Scielo, utilizando-se os seguintes termos selecionados: Ace Polymorphism, Apoe Polymorphism, Enos Polymorphism, Resistance Training, Eccentric Resistance Training, Elderly Women. Os critérios de inclusão para a seleção dos artigos foram: (i) estudos publicados em revistas indexadas; (ii) estudos publicados em inglês e/ou português e/ou espanhol; (iii) estudos realizados com seres humanos do sexo femininos e idosas; (iv) pesquisas originais com intervenção de exercício físico (v) estudos publicados entre 2012 e 2017.

Foram encontrados 31 artigos potencialmente selecionáveis para compor a presente fundamentação e base desta pesquisa. Uma análise inicial foi realizada com base nos títulos dos manuscritos, em seguida, procedeu-se a análise dos resumos, na qual foram 28 excluídos por não estarem de acordo com os critérios de inclusão, como estudos realizados com seres humanos do sexo femininos e idosas, artigos de revisão e/ou meta- análises, não publicados entre 2012 e 2017. Por fim, foram selecionados apenas 3 artigos que se encaixavam nos critérios de inclusão. Destes, apenas um tratava sobre a respostas do polimorfismo do gene em eNOS ao exercício excêntrico em idosas (TEIXEIRA et al., 2015), dois sobre treinamento resisto e as influências do polimorfismo genético de ECA (MOTA et al., 2013; PEREIRA et al., 2013), e nenhum trazia informações sobre polimorfismos de APOE e treinamento.

No estudo de Teixeira et al., (2015), foi realizada uma intervenção de TFE com 87 idosas sedentárias com percentual de gordura elevado, investigando a associação entre o polimorfismo do gene eNOS Glu298Asp com força muscular, composição corporal e variáveis bioquímicas além de investigar a cinética de ON em resposta ao TFE e a possível influência do polimorfismo do gene eNOS nesta resposta. O TFE seguiu o protocolo de Funghetto et al., (2013). O estudo apresentou que ON permaneceu elevado até 48 horas após uma sessão de TFE aguda, sem que houvesse uma interação genotípica. Além disso, foi demonstrado que sujeitos portadores do genótipo GG apresentaram maior peso corporal, prevalência de 
obesidade, IMC e maior força muscular relativa, enquanto que o grupo TT / TG apresentou maiores concentrações de triglicérides, VLDL e ureia, indicando também que os portadores do alelo T tendem para maiores fatores de risco cardiovascular e distúrbios metabólicos.

Em estudo realizado por Mota et al., (2013), 64 idosas hipertensas foram divididas em grupo controle e grupo programa de exercício resistido (PER) desenvolvido durante 4 meses com frequência de 3 vezes semanais. Assim foi verificada uma redução crônica nos valores da pressão arterial em repouso após o PER bem como efeito hipotensor pós exercício em sessões agudas para Pressão Arterial Sistólica (PAS) após o segundo e terceiro meses e para Pressão Arterial Diastólica (PAD) após o segundo e quarto meses. Em relação a ECA, não foram observadas diferenças significativas nos principais efeitos e efeitos de interação entre a Pressão arterial e genótipos, e os padrões crônicos e agudos não foram evidenciados no grupo controle. Com isso, o estudo observou um efeito protetor do PER sobre o sistema cardiovascular, mas sem que houvesse uma influência do polimorfismo ECA I / D em idosas hipertensas.

Já no estudo de Pereira et al., (2013), foram estudadas variações genéticas dos polimorfismos humanos ECA I / D e ACTN3 R577X com objetivo de verificar melhoras no desempenho de potência muscular e capacidade funcional. 139 idosas saudáveis foram submetidas a um PER composto por três sessões de treinamento por semana durante 12 semanas. Foram realizados dois exercícios: salto vertical e o lançamento de medicine-ball. Foi indicado ao final do programa de treinamento um aumento significativo no desempenho de capacidade muscular e funcional além de uma interação significativa entre genótipotreinamento para ambos os polimorfismos em todos os índices de desempenho muscular. Assim o estudo sugere que os genótipos de ECA e ACTN3 (únicos ou combinados) exercem uma influência significativa nos fenótipos musculares de mulheres idosas em resposta ao treinamento de alta velocidade.

A relevância desta pesquisa está, com base nos estudos apresentados sobre a influências dos polimorfismos genéticos na expressão de eNOS e ECA com influências para o sistema cardiovascular, e APOE com respostas no perfil lipídico, e tendo em vistas suas consequências para a saúde humana, além da escassez na literatura de estudos com intervenção do treinamento de força em indivíduos idosos e as variáveis genéticas que predizem determinados declínios de saúde (FRATTINI et al., 2016), produzir um estudo que possa elucidar como essas influências genéticas associadas ao treinamento de força podem 
contribuir para o monitoramento da efetividade e segurança desta prática em mulheres idosas com obesidade.

Considerando o cenário exposto, não cabe dentro de uma abordagem sistêmica, o controle de quaisquer variáveis, sem uma fundamentação que dê suporte ao ato, o que requer que estudos sejam realizados visando identificar possíveis influências genéticas nos polimorfismos de eNOS, ECA e APOE na resposta de marcadores de dano muscular após TFE, de modo que contribuam para o monitoramento da efetividade e segurança desta prática, sobretudo em mulheres idosas com obesidade.

\section{METODOLOGIA}

A presente pesquisa foi de caráter quase experimental e de natureza quantitativa, com população de 60 anos ou mais, com excesso de massa adiposa, residentes na comunidade de Ceilândia e Taguatinga - Brasília/DF - e do sexo feminino. As variáveis avaliadas foram o modelo de TFE, o polimorfismo do gene de eNOS, APOE e ECA, marcador de dano muscular CK e análise do perfil metabólico de Colesterol, LDL, HDL, VLDL e TGL.

Local de realização da pesquisa:

O projeto contou com apoio da universidade católica de Brasília (UCB) e da universidade de Brasília (UNB) que possuem a infra-estrutura ambulatorial no Hospital da Universidade Católica de Brasília e no laboratório da faculdade de educação física da Universidade de Brasília, necessária à realização das análises clínicas das idosas e a aferição da composição corporal de cada voluntária da pesquisa por meio do método de Absortometria Radiológica de Dupla Energia (DEXA). O teste e o treino de força foram realizados no Laboratório de Estudos de Força disponibilizado pelo programa de Graduação em Educação Física.

Amostra:

Participaram do estudo 86 mulheres com idade de 60 anos ou mais e que atenderam aos seguintes critérios de inclusão: caracterizadas com sobrepeso ou obesidade detectado através de avaliação em DEXA, que não possuíssem problemas ortopédicos e articulares que impedissem a realização da atividade física excêntrica ou que pudesse agravar o dano 
osteoarticular apresentado na avaliação ortopédica e funcional, e que concordassem em assinar o TCLE. Foram excluídas aquelas que tinham menos de 60 anos de idade, que não apresentaram índice de massa corporal caracterizado como sobrepeso ou obesidade, as que possuíam alguns problemas ortopédicos e articulares nos membros inferiores e que não assinaram o termo de consentimento livre e esclarecido.

\section{Instrumentos e procedimentos metodológicos:}

Os procedimentos para caracterização da amostra e aplicação dos critérios de inclusão e exclusão foram compostos por: avaliação da composição corporal e avaliação ortopédica anteriormente ao início do programa de treinamento de força excêntrico.

- Avaliação da composição corporal: A análise da composição corporal foi realizada por meio do DEXA. O exame foi realizado em equipamento da marca General Electric-GE, modelo 8548 BX1L, ano 2005, tipo Lunar DPX, com software programa Encore 2005, do Laboratório da Faculdade de Educação Física da Universidade de Brasília. Todas as avaliações foram realizadas pelo mesmo técnico, o qual era treinado para realização desses exames. Para executar o procedimento, as voluntárias retiraram todos os pertences de metais (pulseiras, brincos, anéis, aliança, etc.), posicionaram as participantes em decúbito dorsal sobre a mesa do equipamento, de forma que ficassem totalmente centralizadas em relação às laterais da mesa. As voluntárias foram instruídas a se disporem com os membros inferiores estendidos, sendo utilizada uma fita de velcro para manter os membros inferiores próximos e dar suporte aos pés, de forma que esses ficassem numa angulação de aproximadamente 45으 em relação ao plano vertical. Os membros superiores permaneciam estendidos e posicionados ao longo do corpo, sem que houvesse contato com o tronco. Desta avaliação foram utilizadas as seguintes variáveis: Massa Corporal (MC), Estatura, Índice de Massa Corporal (IMC), percentual de Gordura (\%G), Massa Magra (MG). A obesidade foi determinada por DXA, e os valores percentuais de gordura corporal foram distribuídos de acordo com as recomendações de Bouchard (2009), assumindo-se um ponto de corte maior ou igual a $35 \%$ para mulheres obesas. 
- Avaliação ortopédica: Foi realizada uma avaliação ortopédica e reumatológica por um fisioterapeuta para garantir a integridade do sistema músculo-esquelético das participantes, bem como para certificar que o exercício excêntrico não seria prejudicial ou que alguma disfunção neste sistema interferiria no desempenho do mesmo.

\section{Análise Sanguínea:}

Os procedimentos para extração do material genético, quantificação do perfil lipídico e determinação da CK foram realizado através de coleta sanguínea de $25 \mathrm{ml}$ preferencialmente por meio de punção da veia antecubital em tubos a vácuo. Um dos tubos foi destinado à extração do material genético, outro para a quantificação do perfil lipídico e outro para determinação da CK no momento pré- TFE. Todo material utilizado foi devidamente descartado em recipiente adequado. Todas as voluntárias foram encorajadas a evitar o consumo de tabaco, álcool e cafeína, bem como atividade física incomum para evitar influenciar os parâmetros de análise. Todo o procedimento foi realizado por enfermeira devidamente treinada.

- Análise do perfil metabólico: Foi utilizado coletado tubo com acelerador de coagulação (tampa vermelha) para a coleta do sangue destinado a análise lipémica. 0 sangue foi centrifugado em uma centrifuga $\left(C E L M^{\circledR}\right)$ a 3000 rotações por 15 minutos para separação entre soro e porção celular. Logo após o soro foi transferido para um microtubo (eppendorf ${ }^{\circledR}$ ) e armazenado sob refrigeração até ser transportado ao laboratório por meio de uma pipeta (Gilson ${ }^{\circledR}$ ). O colesterol total, as VLDL e a HDL, bem como os TGL foram quantificados por meio de um espectrofotômetro automático, modelo Cobas Mira Plus Roche ${ }^{\circledast}$, utilizando-se reagentes bioquímicos Labtest ${ }^{\circledR}$. Já as LDLs foram estimadas por meio da fórmula de Friedewald, Levy e Fredrickson (1972). Todos os testes acima foram realizados seguindo métodos clínicos de rotina com reagentes da Boehringer Mannheim (Alemanha).

- Análise genotípica:

eNOS: o DNA total foi isolado a partir de leucócitos do sangue periférico de acordo com procedimentos padrão. Reação em cadeira de polimerase (PCR) foi utilizada para 
determinação da amplificação do gene eNOS (rs1799983) seguida de restrição enzimática específica (PCR-RFLP) de acordo com Pulkkinen et al., 2000 e adaptada para este estudo utilizando primers eNOS-F1 (50AAGGCAGGAGACAGTGGATGGA30) e eNOS-R1 (50CCCAGTCAATCCCTTTGGTGCTCA30), seguindo o protocolo utilizado por Teixeira et al 2015.

APOE: o polimorfismo APOE "2 /" 3 / "4 foi genotipado com a enzima de restrição Hhal de um fragmento de reação em cadeia da polimerase (PCR), seguido de eletroforese em gel de agarose $4 \%$ para visualizar os fragmentos correspondentes a cada alelo (BATALLA et al., 2000; WENHAM et al., 1991; DONOHOE et al, 1999).

ECA: reação em cadeia da polimerase (PCR) foi utilizada para identificação do polimorfismo I / D do gene da ECA, usando os primers de iniciação direta (5' CTGGAGACCACTCCCATCCTTTCT-3') e reversa (5' GATGTGGCCATCACATTCGTCAGAT-3'). Os produtos da amplificação foram separados em eletroforese a $80 \mathrm{~V}$, em gel de agarose 1,0\%, e visualizados em luz ultravioleta na presença de brometo de etídeo. Para identificação dos genótipos foi realizada a visualização dos amplicons, determinados pela presença dos alelos D e/ou I. De acordo com método utilizado previamente na literatura por Shanmugan et al., 1993, Mota, et al, 2013, González et al., 2006, para aumentar a especificidade da genotipagem, uma PCR adicional confirmatória foi realizada com todas as amostras portadoras do genótipo $D / D$, utilizando um par de iniciadores específicos para a inserção, ou seja, amplificador do alelo I. Os iniciadores utilizados foram: (5' TGG GAC CAC AGC GCC CGC CAC TAC 3') e (5' TCG CCAGCC CTC CCA TGC CCA TAA 3').

- Análise de CK: A determinação da CK no sangue foi em dois momentos: Antes do início da execução do EFE e logo após a última série, utilizando-se a metodologia cinéticoespectrofotométrica. Para verificar o dano muscular após o término da sessão de TFE foram coletados $5 \mathrm{ml}$ de dosagem sérica de CK e para análise utilizou-se a metodologia cinético-espectrofotométrica.

\section{Procedimentos experimentais de Atividade Física:}

Antes de iniciar a execução do programa de exercício de força excêntrico, as voluntárias foram submetidas à fase de adaptação ao exercício e avaliação da força máxima, seguindo os procedimentos abaixo descritos: 
- Fase de adaptação ao exercício: Por se tratar de uma população não praticante de exercícios regulares, as voluntárias foram submetidas a uma fase de adaptação ao exercício, para conhecerem de qual exercício se tratava e quais padrões adotados para a execução do mesmo.

- Avaliação de Força Máxima: Foram realizadas avaliações através do teste de 10 repetições máximas (10 RM) na cadeira extensora de forma bilateral seguindo de: aquecimento geral (10 minutos de esteira em intensidade leve), onde as voluntárias foram submetidas a oito repetições com $50 \%$ de 10 RM estimada de acordo com a capacidade de cada participante verificada na sessão de adaptação realizada nos dois dias anteriores, após um minuto de intervalo, foram realizadas três repetições com 70\% de 10 RM estimada. Depois de três minutos as tentativas subsequentes foram realizadas para uma repetição com cargas progressivamente mais pesadas até que a 10 RM foi determinada em três tentativas, utilizando três minutos de descanso entre as tentativas. As padronizações das angulações e movimentos dos exercícios foram conduzidas de acordo com as descrições de Brown e Weir (2001). Para certificar que as 10 RM pré-treinamento foram ajustadas antes do início do treinamento a correlação intra-classe foi determinada entre a segunda e a terceira tentativa do teste de 10 RM. A maior 10 RM determinada a partir das últimas duas tentativas foi a utilizada como medida inicial.

- Aplicação do TFE: Após dois dias de descanso as voluntárias foram submetidas a uma série de aquecimento geral em cicloergômetro de membros inferiores durante 10 minutos a $60 \mathrm{rpm}$ e $50 \mathrm{w}$, seguido de aquecimento especifico de 10 repetições a $50 \%$ de $10 \mathrm{RM}$, com intervalo de 3 minutos no mesmo horário em que fizeram o treino de avaliação. A sessão de treino na cadeira extensora bilateral foi realizada $110 \%$ de 10 RM, somente na fase excêntrica, sendo a fase concêntrica executada pelo avaliador. Foram utilizadas sete séries de 10 repetições com descanso passivo de 3 minutos entre as séries e com intervalos de 15 segundos entre cada repetição de 2 segundos. $O$ treino excêntrico foi adaptado de Willoughby e McFarlin (2003). As participantes do estudo foram orientadas a realizar a extensão dos joelhos mesmo que não conseguissem vencer a resistência. 


\section{Análise Estatística}

Todas as análises foram realizadas utilizando o Pacote Estatístico para Ciências Sociais (IBM SPSS, IBM Corporation, Armonk, NY, EUA, 25.0). Teste de Shapiro-Wilk foi utilizado para verificar a distribuição de normalidade dos dados. Para comparação das características dos grupos por genótipos foi utilizado teste t para amostras independentes quando verificada normalidade e teste $U$ de Mann-Whitney quando em distribuição anormal. Análise de variância (ANOVA Fatorial de delineamento misto) 2x4 (genótipo x momento) foi empregada para avaliar a resposta de CK nos genótipos de cada polimorfismo nos quatro momentos (Pré, Pós 0, Pós 3hrs, Pós 24hrs). Tratamento de Bonferroni foi utilizado para identificar as diferenças significativas. Adotou-se $p \leq 0,05$ como nível de significância.

\section{Preceitos éticos}

Este projeto foi submetido ao Comitê de Ética em Pesquisa da Secretaria de Estado de Saúde do Distrito Federal e aprovado com o número de protocolo 035/2011.

\section{RESULTADOS E DISCUSSÃO}

Participaram do presente estudo 86 mulheres $(69,24 \pm 4,94$ anos) caracterizadas com obesidade > 35\% gordura corporal. Na Tabela 1 estão apresentados os parâmetros antropométricos, bioquímicos e de força das idosas sem considerar os genótipos, a fim de caracterizar a amostra estudada.

Especificamente, as voluntárias do presente estudo demonstraram elevado percentual de gordura (LOHMAN, 1992) e dislipidemia caracterizados pelas médias elevadas e próximas ao limite superior dos valores de normalidade (SILVA et al., 2012; XAVIER, et al., 2013), além de IMC elevado com valores descritos por Lipschitz (1994) e ABESO (2016), mesmo este, segundo a Associação Brasileira para o Estudo da Obesidade e da Síndrome Metabólica, não distinguindo massa gordurosa de massa magra, podendo ser menos preciso em indivíduos mais idosos, pode ser simples, prático e de baixo custo (ABESO, 2016) (Tabela 1). 
Tabela 1. Caracterização dos parâmetros antropométricos, metabólicos e de força da amostra - Brasília, 2018.

\begin{tabular}{lcc}
\hline & Média & \pm DP \\
\hline Massa Corporal (kg) & 63,23 & 8,96 \\
Estatura (m) & 1,53 & 0,06 \\
IMC (Kg/m²) & 27,38 & 3,70 \\
\%G (\%) & 41,61 & 5,60 \\
MM (Kg) & 35,62 & 3,34 \\
CTotal (mg/dL) & 206,76 & 39,11 \\
HDL (mg/dL) & 47,07 & 11,15 \\
LDL (mg/dL) & 131,61 & 32,57 \\
VLDL (mg/dL) & 28,59 & 12,77 \\
TGL (mg/dL) & 141,13 & 63,08 \\
CK (U/L) & 91,69 & 32,81 \\
FM - 10 RM (kg) & 36,60 & 9,29 \\
\hline
\end{tabular}

IMC= índice de massa corporal. \%G= percentual de gordura. $\mathrm{MM}=$ massa magra. CTotal= colesterol total. $\mathrm{HDL}=$ lipoproteína de alta densidade. $L D L=$ lipoproteína de baixa densidade. $V L D L=$ lipoproteína de muito baixa densidade. $T G L=$ triglicerídeo. $\mathrm{FM}-10 \mathrm{RM}=$ Força máxima -10 repetições máximas.

Para efeitos de descrição, na Tabela 2 estão os dados referentes as frequências absolutas e relativas de alelos dos genótipos para eNOS, APOE e ECA na amostra estudada.

Tabela 2. Frequências genotípicas absolutas e relativas de eNOS, APOE e ECA da amostra Brasília, 2018.

\begin{tabular}{lccc}
\hline & Genótipo & FA ( $\mathbf{n}=\mathbf{8 0})$ & FR (\%) \\
\hline eNOS & GG & 33 & $38,37 \%$ \\
& TG & 47 & $54,65 \%$ \\
APOE & TT & 6 & $6,98 \%$ \\
& e3/e3 & 64 & $74,42 \%$ \\
& e3/e4 & 19 & $22,09 \%$ \\
ECA & e2/e3 & 3 & $3,49 \%$ \\
& DD & 36 & $41,86 \%$ \\
& DI & 31 & $36,05 \%$ \\
& II & 19 & $22,09 \%$ \\
\hline
\end{tabular}

$\mathrm{FA}=$ frequência absoluta. $\mathrm{FR}=$ frequência relativa em percentual. 
Considerando a frequência muito baixa de portadores homozigóticos TT, estes foram agrupados com portadores TG heterozigóticos como descrito por Teixeira et al 2015. Para APOE, apenas 3 voluntárias apresentaram o genótipo e2/e3, e para efeitos desta análise, foram agrupadas com aquelas que apresentaram genótipo e3/34. Tendo em vista que apenas 17 voluntárias apresentaram genótipo II, optou-se pela união dos genótipos II e ID para uma adequada comparação entre os grupos genotípicos, assim como realizado em estudos anteriores de Fernandes et al., (2015), Pescatello et al., (2007), Santana et al., (2011).

Nas tabelas 3, 4 e 5 estão os dados de caracterização dos padrões antropométricos, metabólicos e de força da amostra estuda, divididos e comparados por grupo de genótipo de eNOS, APOE e ECA respectivamente.

Para o polimorfismo de eNOS Glu298Asp, entre os genótipos GG e TG/TT não foram observadas diferenças estatisticamente significativas para nenhuma das variáveis de caracterização antropométrica, perfil metabólico ou de força ( $p>0,05$ ) (Tabela 3). Diferentemente, Teixeira et al., (2015) demonstraram que o genótipo GG apresentava maior peso corporal, maior prevalência de obesidade, IMC e maior força muscular relativa, em relação ao genótipo TG/TT, enquanto TGL e VLDL foram significativamente menores para GG.

Tabela 3. Comparação das características da amostra de acordo com genótipo de eNOS Brasília, 2018.

\begin{tabular}{lccc}
\hline & GG (n=33) & TG/TT (n=53) & p \\
\hline Massa Corporal (kg) & $63,53 \pm 10,00$ & $63,04 \pm 8,37$ & $0,806^{\text {a }}$ \\
Estatura (m) & $1,52 \pm 0,06$ & $1,53 \pm 0,06$ & $0,444^{\text {a }}$ \\
IMC (Kg/m²) & $27,49 \pm 4,41$ & $27,32 \pm 2,23$ & $0,844^{\text {a }}$ \\
\%G (\%) & $40,43 \pm 6,26$ & $42,35 \pm 5,08$ & $0,122^{\text {a }}$ \\
MM (Kg) & $36,15 \pm 3,87$ & $35,29 \pm 2,95$ & $0,246^{\text {a }}$ \\
CTotal (mg/dL) & $209,79 \pm 35,52$ & $204,87 \pm 41,40$ & $0,908^{\mathrm{b}}$ \\
HDL (mg/dL) & $45,94 \pm 10,45$ & $47,79 \pm 11,61$ & $0,351^{\mathrm{b}}$ \\
LDL (mg/dL) & $133,83 \pm 32,12$ & $130,22 \pm 33,07$ & $0,620^{\mathrm{a}}$ \\
VLDL (mg/dL) & $28,22 \pm 13,21$ & $28,81 \pm 12,61$ & $0,786^{\mathrm{b}}$ \\
TGL (mg/dL) & $137,03 \pm 62,50$ & $143,68 \pm 63,91$ & $0,619^{\mathrm{b}}$ \\
FM - 10 RM (kg) & $37,81 \pm 8,14$ & $35,85 \pm 9,95$ & $0,343^{\mathrm{a}}$ \\
\hline
\end{tabular}

a valor de $\mathrm{p}$ identificado por teste $\mathrm{t}$ independente. ${ }^{\mathrm{b}}$ valor de $\mathrm{p}$ identificado por este $\mathrm{U}$ de Mann-Whitney. 
Entre os genótipos de APOE e3/e3, e3/e4-e2/e3 identificados na amostra não foram observadas diferenças estatisticamente significativas para nenhuma das variáveis de caracterização antropométrica, perfil metabólico ou de força $(p>0,05)$ (Tabela 4). Em jovens, Freitas et al., (2015) encontraram o genótipo e3/e3 mais presente para uma ou mais variável lipídica alterada, porém assim como no presente estudo, este genótipo mostrou um número maior de voluntários. Além disso o genótipo e2e3 estavam alocados no grupo que apresentavam características de variáveis lipídicas analisadas (CTotal, TGL, HDL e LDL) normais nas duas avaliações realizadas.

Diferente do presente estudo, que não encontrou diferenças significativas entre os genótipos de APOE, em estudo realizado por Martins et al., (2008) com 672 indivíduos, com idade entre 18 a 80 anos, foram observados menores níveis de HDL e colesterol em portadores do genótipo e3e4 em relação aos genótipos e3e3 e e2e3.

$\mathrm{Na}$ amostra do presente estudo, não foram avaliados possíveis casos de DM, porém estudo de Zhen et al., (2018) demonstram que idosos com DM tipo 2 com a presença do alelo e4 possuem maior concentração sérica de TGL comparados aos portadores do alelo e2 e e3. Além disto, indivíduos diabéticos portadores de e4 e e2 apresentaram maior nível de TGL em comparação com indivíduos não diabéticos.

Tabela 4. Comparação das características da amostra de acordo com genótipo de APOE Brasília, 2018.

\begin{tabular}{lccc}
\hline & e3/e3 (n=64) & e3/e4-e2/e3 (n=22) & p \\
\hline Massa Corporal (kg) & $62,58 \pm 8,39$ & $65,11 \pm 10,45$ & $0,256^{\text {a }}$ \\
Estatura (m) & $1,52 \pm 0,06$ & $1,54 \pm 0,06$ & $0,122^{\text {a }}$ \\
IMC (Kg/m²) & $27,17 \pm 3,57$ & $28,01 \pm 4,08$ & $0,359^{\text {a }}$ \\
\%G (\%) & $41,26 \pm 5,77$ & $42,63 \pm 5,07$ & $0,327^{\text {a }}$ \\
MM (Kg) & $35,55 \pm 3,21$ & $35,82 \pm 3,76$ & $0,737^{\text {a }}$ \\
CTotal (mg/dL) & $204,13 \pm 37,63$ & $214,41 \pm 43,14$ & $0,839^{\mathrm{b}}$ \\
HDL (mg/dL) & $47,01 \pm 11,65$ & $47,27 \pm 9,81$ & $0,579^{\mathrm{b}}$ \\
LDL (mg/dL) & $129,07 \pm 29,11$ & $139,00 \pm 40,92$ & $0,596^{\mathrm{b}}$ \\
VLDL (mg/dL) & $28,74 \pm 13,09$ & $28,14 \pm 12,06$ & $0,847^{\mathrm{b}}$ \\
TGL (mg/dL) & $141,36 \pm 64,49$ & $140,45 \pm 60,27$ & $0,957^{\mathrm{b}}$ \\
FM - 10 RM (kg) & $37,25 \pm 9,67$ & $34,71 \pm 8,00$ & $0,271^{\mathrm{a}}$ \\
\hline
\end{tabular}

${ }^{a}$ valor de $p$ identificado por teste $t$ independente. ${ }^{b}$ valor de $p$ identificado por este $U$ de Mann-Whitney. 
Tabela 5. Comparação das características da amostra de acordo com genótipo de ECA Brasília, 2018.

\begin{tabular}{lccc}
\hline & DD (n=36) & DI/II (n=50) & $p$ \\
\hline Massa Corporal (kg) & $64,13 \pm 9,45$ & $62,58 \pm 8,64$ & $0,431^{\text {a }}$ \\
Estatura (m) & $1,52 \pm 0,06$ & $1,53 \pm 0,06$ & $0,688^{\text {a }}$ \\
IMC (Kg/m²) & $28,10 \pm 4,21$ & $26,87 \pm 3,24$ & $0,127^{\text {a }}$ \\
\%G (\%) & $41,73 \pm 4,85$ & $41,53 \pm 6,14$ & $0,869^{\text {a }}$ \\
MM (Kg) & $36,10 \pm 2,49$ & $35,27 \pm 3,82$ & $0,256^{\text {a }}$ \\
CTotal (mg/dL) & $211,47 \pm 30,78$ & $203,36 \pm 44,14$ & $0,255^{\mathrm{b}}$ \\
HDL (mg/dL) & $44,58 \pm 10,25$ & $48,87 \pm 11,53$ & $0,060^{\mathrm{b}}$ \\
LDL (mg/dL) & $134,69 \pm 26,23$ & $129,39 \pm 36,56$ & $0,460^{\mathrm{a}}$ \\
VLDL (mg/dL) & $31,42 \pm 13,88$ & $26,54 \pm 11,62$ & $0,074^{\mathrm{b}}$ \\
TGL (mg/dL) & $153,67 \pm 67,42$ & $132,10 \pm 58,80$ & $0,109^{\mathrm{b}}$ \\
FM - 10 RM (kg) & $38,06 \pm 9,52$ & $35,55 \pm 9,07$ & $0,308^{\mathrm{b}}$
\end{tabular}

a valor de $\mathrm{p}$ identificado por teste $\mathrm{t}$ independente. ${ }^{\mathrm{b}}$ valor de $\mathrm{p}$ identificado por este $\mathrm{U}$ de Mann-Whitney. ${ }^{*}$ Diferença estatisticamente significativa em relação ao genótipo $\mathrm{DD}(p \leq 0,05)$.

Assim como nos outros polimorfismos aqui estudados, para os genótipos da ECA não foram observadas diferenças estatisticamente significativas para nenhuma das variáveis de perfil antropométrico, metabólico ( $p>0,05)$ (Tabela 5). Diferentemente, Lima et al., (2011) demonstraram que idosas portadoras do genótipo $D / D$ apresentaram maior massa livre de gordura relativa em comparação aos portadores do alelo I.

Relacionado a força, no presente estudo não foram encontradas diferenças significativas $(p>0,05)$. Com um outro método de avaliação de força, através da força de preensão manual, Frattini et al., (2016) verificaram não haver uma influência genotípica de ECA para força muscular, assim como em mulheres de meia idade, em estudo de Tibana et al., (2014), não foi demonstrado um papel importante dos genes da ECA na determinação de força, volume e qualidade muscular. No entanto, Charbonneau et al., (2008) verificaram que os portadores do genótipo DD exibiram volume muscular significativamente maior em comparação com os portadores do genótipo II. 
Tabela 6. Concentração de CK pré e pós TFE distribuídos por genótipos de eNOS, APOE e ECA - Brasília, 2018.

\begin{tabular}{|c|c|c|c|c|}
\hline & CK Pré & CK Pós0 & CK Pós3hrs & CK Pós24hrs \\
\hline \multicolumn{5}{|l|}{ eNOS } \\
\hline GG & $87,76 \pm 30,98$ & $90,94 \pm 36,29$ & $89,85 \pm 34,74$ & $95,61 \pm 39,35$ \\
\hline $\mathrm{TG} / \mathrm{TT}$ & $94,13 \pm 33,96$ & $90,85 \pm 36,65$ & $91,75 \pm 35,58$ & $107,74 \pm 42,35^{\dagger \mp}$ \\
\hline \multicolumn{5}{|l|}{ APOE } \\
\hline e3/e3 & $91,77 \pm 33,02$ & $90,81 \pm 35,08$ & $89,58 \pm 35,00$ & $105,22 \pm 43,70^{\dagger \mp}$ \\
\hline e3/e4-e2/e3 & $91,45 \pm 32,98$ & $91,09 \pm 40,51$ & $95,23 \pm 36,05$ & $96,86 \pm 34,03$ \\
\hline \multicolumn{5}{|l|}{ ECA } \\
\hline $\mathrm{DD}$ & $96,97 \pm 32,24$ & $94,53 \pm 33,71$ & $94,33 \pm 33,97$ & $100,75 \pm 34,03$ \\
\hline DI/II & $87,88 \pm 33,01$ & $88,26 \pm 38,18$ & $88,64 \pm 35,99$ & $104,76 \pm 46,27^{\#+\mp}$ \\
\hline
\end{tabular}

A concentração da CK nas condições Pré-intervenção e momentos pós-exercício não foi significativamente diferente entre os genótipos GG e TG/TT ( $p>0,05)$. O TFE induziu alterações significativas na concentração de CK apenas para o genótipo TG/TT ao longo do tempo, aumentando significativamente no momento Pós24hrs em relação aos momentos Pós0 e Pós3hrs ( $p=0,006)$, de 90,85 $\pm 36,65 \mathrm{U} / \mathrm{I}$ e 91,75 $\pm 35,58 \mathrm{U} / \mathrm{I}$ respectivamente para $107,74 \pm$ 42,35 U/I Pós24hrs.

A concentração da CK nas condições Pré-intervenção e momentos pós-exercício não foi significativamente diferente $(p>0,05)$ entre os genótipos e3/e3 e e3/e4-e2/e3. Foram observadas alterações significativas na concentração de CK apenas para o genótipo e3/e3 ao longo do tempo, com um aumento significativo de 90,81 \pm 35,08 U/I Pós0, 89,58 \pm 35,00 U/I Pós3hrs para 105,22 $\pm 43,70 \mathrm{U} / \mathrm{I}$ no momento Pós $24 \mathrm{hrs}(\mathrm{p}=0,013 ; \mathrm{p}=0,002)$ respectivamente.

Para o polimorfismo de ECA, a concentração da CK nas condições Pré-intervenção e momentos pós-exercício não foi significativamente diferente entre os genótipos DD e DI/II (p $>0,05)$. O TFE induziu alterações significativas na concentração de CK apenas para o genótipo DI/II ao longo do tempo, com um aumento significativamente de 87,88 $\pm 33,01 \mathrm{U} / \mathrm{I}$ Pré ( $\mathrm{p}=$ 0,042), 88,26 \pm 3 8,18UI Pós0 ( $p=0,011$ ) e 88,64 $\pm 35,99$ Pós3hrs ( $p=0,008$ ) respectivamente ao momento Pós24hrs 104,76 \pm 46,27 U/I. Porém, em estudo, Pereira et al., (2013) após 12 semanas de treinamento de força de alta velocidade demonstraram que idosas com ambos 
os genótipos ID e DD aumentaram significativamente força máxima em 1RM para supino, potência e capacidade funcional do que indivíduos com o genótipo II.

Para os polimorfismos de eNOS, APOE e ECA, não são encontrados na literatura dados referentes a interação genotípica destes com a resposta de CK após treinamento em idosos. Os níveis aumentados de CK no presente estudo a partir do momento 24hrs após TFE, parecem ir de encontro com dados observado em estudos anteriores como de Funghetto et al., (2013) observaram a influência genotípica na resposta de CK com aumentos a partir de 24hrs após TFE porém através de polimorfismos de IL-6. Além disso, Tajra et al., (2014) também observaram em idosas obesas, aumento na CK e IL-6 após 48hrs e 24hrs após sessão de TFE, porém tais níveis foram considerados normais e não indicativos de excesso de dano muscular assim como no presente estudo. E estudo de Teixeira et al., (2015), indicando que o protocolo de TFE utilizado é suficiente para induzir dano muscular pela mediado através da CK, mas sem uma resposta exacerbada.

Não houve uma diferença estatisticamente significativa entre os genótipos para a resposta de CK dos polimorfismos de eNOS, APOE e ECA. O que possivelmente explica a não interação entre os genótipos, se dá ao fato que todos as voluntárias que reportaram genótipos diferentes realizaram a mesma metodologia de treino, o TFE, e é sabido que dentre os três tipos de contrações, as excêntricas induzem mais danos aos músculos em comparação com os tipos de contração concêntrica e isométrica (WESTING et al., 1991). Estudos posteriores comparando outros métodos de treinamento para interação entre genótipos pode ser de grande relevância.

Cabe ressaltar as principais limitações do presente estudo, como o não monitoramento de variáveis metabólicas e hemodinâmicas nos momentos Pré e Pós TFE assim como de outros marcadores de dano muscular e em mais momentos pós treino.

\section{CONCLUSÃO}

Os genótipos dos polimorfismos de eNOS, APOE e ECA parecem não influenciar para no perfil antropométrico, metabólico possivelmente porque todas as mulheres apresentaram estes elevados aos valores de normalidade. Assim também não houve diferença significativa entre os genótipos destes polimorfismos para força muscular e diferenças para resposta de 
CK. Porém o protocolo de TFE pareceu induzir um aumento de CK a partir de 24 horas após o exercício para os genótipos TG/TT de eNOS, e3/e3 de APOE e DI/II de ECA sem interação entre os genótipos.

Demais estudos com utilização de outros biomarcadores de dano muscular e em mais momentos, assim como o monitoramento de respostas metabólicas e hemodinâmicas para verificar a interação dos genótipos sobre estas variáveis são de extrema importância. Além de estudos que demonstrem a associação da CK com a reposta inflamatória e a possível influência dos polimorfismos de eNOS, APOE e ECA em idosas obesas.

\section{Referências}

Associação Brasileira para o Estudo da Obesidade e da Síndrome Metabólica Diretrizes brasileiras de obesidade 2016 / ABESO - Associação Brasileira para o Estudo da Obesidade e da Síndrome Metabólica. - 4.ed. - São Paulo, SP

American college of sports medicine (ACSM). Diretrizes do ACSM para os testes de esforço e sua prescrição. 7. ed. Rio de Janeiro: Guanabara Koogan, 2007.

ANDREW, PJ.; MAYER, B. Enzymatic function of nitric oxide synthases. Cardiovascular research, v. 43, n. 3, p. 521-531, 1999.

ASGHAR, M. et al. Prevalence of MTHFR, Factor V, ACE and APOE gene polymorphisms among Muslims of Manipur, India. Annals of human biology, v. 40, n. 1, p. 83-87, 2013.

BAIRD, Marianne F. et al. Creatine-kinase-and exercise-related muscle damage implications for muscle performance and recovery. Journal of nutrition and metabolism, v. 2012, 2012.

BALSAMO, S.; SIMÃO, R. Treinamento de força para osteoporose, fibromialgia, diabetes tipo 2, artrite reumatóide e envelhecimento. Phorte, 2007.

BATALLA, Alberto et al. Synergistic effect between apolipoprotein $E$ and angiotensinogen gene polymorphisms in the risk for early myocardial infarction. Clinical chemistry, v. 46, $n$. 12, p. 1910-1915, 2000.

BAUMAN, A. et al. Updating the evidence for physical activity: summative reviews of the epidemiological evidence, prevalence, and interventions to promote "Active Aging". The Gerontologist, v. 56, n. Suppl 2, p. S268-S280, 2016.

BELL, Christopher G.; WALLEY, Andrew J.; FROGUEL, Philippe. The genetics of human obesity. Nature reviews genetics, v. 6, n. 3, p. 221, 2005. 
BOUCHARD, Danielle R.; DIONNE, Isabelle J.; BROCHU, Martin. Sarcopenic/obesity and physical capacity in older men and women: data from the Nutrition as a Determinant of Successful Aging (NuAge) - the Quebec Longitudinal Study. Obesity, v. 17, n. 11, p. 20822088, 2009.

BROWN, Lee E.; WEIR, Joseph P. ASEP procedures recommendation I: accurate assessment of muscular strength and power. Journal of Exercise Physiology Online, v. 4, n. 3, 2001.

CAMARANO, Ana Amélia Coordenação. Características das instituições de longa permanência para idosos: região Centro-Oeste. 2008.

CAVALCANTI, C.L. et al. Envelhecimento e obesidade: um grande desafio no século XXI. Revista Brasileira de Ciências da Saúde, v. 14, n. 2, p. 87-92, 2011.

CHANNON, K. M.; GUZIK, T. J. Mechanisms of superoxide production in human blood. Journal of Physiology and Pharmacology, v. 53, n. 4, p. 515-524, 2002.

CHARBONNEAU, D. E. et al. ACE genotype and the muscle hypertrophic and strength responses to strength training. Medicine and science in sports and exercise, v. 40, n. 4, p. 677, 2008.

CHODZKO-ZAJKO, W. J. et al. Exercise and physical activity for older adults. Medicine \& science in sports \& exercise, v. 41, n. 7, p. 1510-1530, 2009.

COLOMBO, M. G. et al. Evidence for association of a common variant of the endothelial nitric oxide synthase gene (Glu298 $\rightarrow$ Asp polymorphism) to the presence, extent, and severity of coronary artery disease. Heart, v. 87, n. 6, p. 525-528, 2002.

COOPER, M. H. et al. Conjugated linoleic acid isomers have no effect on atherosclerosis and adverse effects on lipoprotein and liver lipid metabolism in apoE-/- mice fed a highcholesterol diet. Atherosclerosis, v. 200, n. 2, p. 294-302, 2008.

DONOHOE, Gerard G. et al. Rapid identification of apolipoprotein E genotypes by multiplex amplification refractory mutation system PCR and capillary gel electrophoresis. Clinical chemistry, v. 45, n. 1, p. 143-146, 1999.

DURAND, R. J. et al. Hormonal responses from concentric and eccentric muscle contractions. Medicine \& Science in Sports \& Exercise, v. 35, n. 6, p. 937-943, 2003.

FARACI, F. M.; BRIAN, J. E. Nitric oxide and the cerebral circulation. Stroke, v. 25, n. 3, p. 692703, 1994.

FERNANDES, Manuella de Oliveira et al. Influência do polimorfismo I/D do gene da ECA na HPE de jovens normotensos. Revista brasileira de Medicina do Esporte, v. 21, n. 4, p. 308312, 2015. 
FIORE, E. G. et al. Perfil nutricional de idosos freqüentadores de unidade básica de saúde. Rev Ciênc Méd (Campinas), v. 15, n. 5, p. 369-77, 2006.

FÖRSTERMANN, U.; BOISSEL, J.; KLEINERT, H. Expressional control of the 'constitutive'isoforms of nitric oxide synthase (NOS I and NOS III). The FASEB Journal, v. 12, n. 10, p. 773-790, 1998.

FOWKES, F. G. R. et al. Methylene tetrahydrofolate reductase (MTHFR) and nitric oxide synthase (ecNOS) genes and risks of peripheral arterial disease and coronary heart disease: Edinburgh Artery Study. Atherosclerosis, v. 150, n. 1, p. 179-185, 2000.

FRATTINI, I. R. et al. Associação de polimorfismos genéticos da ECA e da ACTN3 com capacidade funcional e prevalência de quedas em mulheres no final da idade adulta e início da terceira idade. Journal of Physical Education, v. 27, n. 1, p. 2713, 2016.

FREITAS, R. G. A. et al. Polimorfismos dos Genes APOE e RLDL e Tracking de Dislipidemia em Jovens. Estudo do Rio de Janeiro. Arq Bras Cardiol, v. 104, n. 6, p. 468-475, 2015.

FRIEDEWALD, William T.; LEVY, Robert I.; FREDRICKSON, Donald S. Estimation of the concentration of low-density lipoprotein cholesterol in plasma, without use of the preparative ultracentrifuge. Clinical chemistry, v. 18, n. 6, p. 499-502, 1972.

FUNGHETTO, S. S. et al. Interleukin-6-174G/C gene polymorphism affects muscle damage response to acute eccentric resistance exercise in elderly obese women. Experimental gerontology, v. 48, n. 11, p. 1255-1259, 2013.

GONZÁLEZ, Antonio J. et al. ACE gene polymorphism and erythropoietin in endurance athletes at moderate altitude. Medicine and science in sports and exercise, v. 38, n. 4, p. 688-693, 2006.

GORDON, S. E. et al. ANG II is required for optimal overload-induced skeletal muscle hypertrophy. American Journal of Physiology-Endocrinology And Metabolism, v. 280, n. 1, p. E150-E159, 2001.

GRUNDY, S. M. Obesity, metabolic syndrome, and coronary atherosclerosis. 2002.

HALPERN, Al.; MANCINI, M. C. Manual de obesidade para o clínico. Editora Roca, 2002.

HAYFLINCK, L. Como e por que envelhecemos. Rio de Janeiro: campus, 1996.

HERNÁNDEZ, D. et al. The ACE/DD genotype is associated with the extent of exerciseinduced left ventricular growth in endurance athletes. Journal of the American College of Cardiology, v. 42, n. 3, p. 527-532, 2003.

ICHIHARA, S. et al. Association of a polymorphism of the endothelial constitutive nitric oxide synthase gene with myocardial infarction in the Japanese population. The American journal of cardiology, v. 81, n. 1, p. 83-86, 1998. 
KRAEMER, W. J. et al. The influence of muscle action on the acute growth hormone response to resistance exercise and short-term detraining. Growth Hormone \& IGF Research, v. 11, n. 2, p. 75-83, 2001.

LEE, Y. H. et al. Associations between eNOS polymorphisms and susceptibility to systemic lupus erythematosus: a meta-analysis. Inflammation Research, v. 61, n. 2, p. 135-141, 2012.

LIMA, R. M. et al. ACE and ACTN3 genotypes in older women: muscular phenotypes. International journal of sports medicine, v. 32, n. 1, p. 66, 2011.

LIPSCHITZ, David A. Screening for nutritional status in the elderly. Primary care, v. 21, n. 1, p. 55-67, 1994.

LOHMAN, Timothy G. Advances in body composition assessment. Human Kinetics, p. 1-23, 1992.

MARTINELLI, Nicola et al. Additive effect of LRP8/APOER2 R952Q variant to APOE $\varepsilon 2 / \varepsilon 3 / \varepsilon 4$ genotype in modulating apolipoprotein $E$ concentration and the risk of myocardial infarction: a case-control study. BMC medical genetics, v. 10, n. 1, p. 41, 2009.

MARTINS, M. C. et al. Influência dos Polimorfismos da APOE em alguns Fatores de risco de Aterosclerose. Acta Médica Portuguesa, v. 21, p. 433-440, 2008.

MATSUDO, S. M. M. Evolução da aptidão física e capacidade funcional de mulheres ativas acima de 50anos de idade de acordo com a idade cronologica. 2001.

MENEZES, T. N. et al. Perfil antropométrico dos idosos residentes em Campina GrandePB. Revista Brasileira de Geriatria e Gerontologia, v. 16, n. 1, p. 19-27, 2013.

MONTGOMERY, H. E. et al. Human gene for physical performance. Nature, v. 393, n. 6682, p. 221-222, 1998.

MOTA, M. R. et al. Acute and chronic effects of resistance exercise on blood pressure in elderly women and the possible influence of ACE I/D polymorphism. Int J Gen Med, v. 6, n. 581-7, p. 6, 2013.

PEDERSEN, B.K. IL-6 signalling in exercise and disease. Biochem Soc Trans., v. 35, p. 1295-7, 2007.

PEREIRA, A. et al. The influence of ACE ID and ACTN3 R577X polymorphisms on lowerextremity function in older women in response to high-speed power training. BMC geriatrics, v. 13, n. 1, p. 131, 2013.

PESCATELLO, Linda S. et al. Dietary calcium intake and renin angiotensin system polymorphisms alter the blood pressure response to aerobic exercise: a randomized control design. Nutrition \& metabolism, v. 4, n. 1, p. 1, 2007. 
PETERSEN A.M.W.; PEDERSEN B. K. The role of IL- 6 in mediating the anti-inflammatory effects of exercise. J Physiol Pharmacol., v. 10, p. 43-51, 2006.

PETROVAN, R. J. et al. DNA Vaccination Against VEGF Receptor 2 Reduces Atherosclerosis in LDL Receptor-Deficient Mice. Arteriosclerosis, thrombosis, and vascular biology, v. 27, n. 5, p. 1095-1100, 2007.

PULKKINEN, Arto et al. Intron 4 polymorphism of the endothelial nitric oxide synthase gene is associated with elevated blood pressure in type 2 diabetic patients with coronary heart disease. Journal of molecular medicine, v. 78, n. 7, p. 372-379, 2000.

PURKAYASTHA, S. et al. Surface electromyographic amplitude-to-work ratios during isokinetic and isotonic muscle actions. Journal of athletic training, v. 41, n. 3, p. 314, 2006.

RAUEN, M. S. et al. Avaliação do estado nutricional de idosos institucionalizados. Revista de Nutrição, v. 21, n. 3, p. 303-310, 2008.

REMAUD, A.; CORNU, C.; GUÉVEL, A. A methodologic approach for the comparison between dynamic contractions: influences on the neuromuscular system. Journal of athletic training, v. 40, n. 4, p. 281, 2005.

RIGAT, B. et al. An insertion/deletion polymorphism in the angiotensin I-converting enzyme gene accounting for half the variance of serum enzyme levels. Journal of Clinical Investigation, v. 86, n. 4, p. 1343, 1990.

ROIG, M. et al. Preservation of eccentric strength in older adults: Evidence, mechanisms and implications for training and rehabilitation. Experimental gerontology, v. 45, n. 6, p. 400-409, 2010.

RUSH, J. W. E.; DENNISS, S. G.; GRAHAM, D. A. Vascular nitric oxide and oxidative stress: determinants of endothelial adaptations to cardiovascular disease and to physical activity. Canadian journal of applied physiology, v. 30, n. 4, p. 442-474, 2005.

SANTANA, Hugo AP et al. The higher exercise intensity and the presence of allele I of ACE gene elicit a higher post-exercise blood pressure reduction and nitric oxide release in elderly women: an experimental study. BMC Cardiovascular disorders, v. 11, n. 1, p. 71, 2011.

SCHMITZ, R. J.; WESTWOOD, K. C. Knee extensor electromyographic activity-to-work ratio is greater with isotonic than isokinetic contractions. Journal of athletic training, v. 36, n. 4, p. 384, 2001.

SHANMUGAM, Vedapuri; SELL, Kenneth W.; SAHA, Bratin K. Mistyping ACE

heterozygotes. Genome Research, v. 3, n. 2, p. 120-121, 1993. 
SILVA, Alessandro O. et al. Comparison of inflammatory, metabolic, and anthropometric parameters in elderly women with and without insulin resistance. Research on Aging, v. 34, n. 3, p. 261-274, 2012.

SMITH, Jonathan D. Apolipoprotein E4: an allele associated with many diseases. Annals of medicine, v. 32, n. 2, p. 118-127, 2000.

SOUBRIER, F. From an ACE polymorphism to genome-wide searches for eQTL. The Journal of clinical investigation, v. 123, n. 1, p. 111-112, 2013.

STRASSER, Barbara; SCHOBERSBERGER, Wolfgang. Evidence for resistance training as a treatment therapy in obesity. Journal of obesity, v. 2011, 2010.

TAJRA, Vitor et al. Identification of high responders for interleukin- 6 and creatine kinase following acute eccentric resistance exercise in elderly obese women. Journal of science and medicine in sport, v. 17, n. 6, p. 662-666, 2014.

TEIXEIRA, T. G. et al. Endothelial nitric oxide synthase Glu298Asp gene polymorphism influences body composition and biochemical parameters but not the nitric oxide response to eccentric resistance exercise in elderly obese women. Clinical physiology and functional imaging, 2015.

TIBANA, R. A. et al. Dissociação do polimorfismo do gene da enzima conversora de angiotensina com a força, volume e qualidade muscular em mulheres sedentárias. ConScientiae Saúde, v. 13, n. 3, p. 411-420, 2014.

WENHAM, PhilipR; PRICE, WilliamH; BLUNDELL, Gillian. Apolipoprotein E genotyping by onestage PCR. The Lancet, v. 337, n. 8750, p. 1158-1159, 1991.

WESTERKAMP, C. M.; GORDON, S. E. Angiotensin-converting enzyme inhibition attenuates myonuclear addition in overloaded slow-twitch skeletal muscle. American Journal of Physiology-Regulatory, Integrative and Comparative Physiology, v. 289, n. 4, p. R1223R1231, 2005.

WILLOUGHBY, D. S.; MCFARLIN, B.; BOIS, C. Interleukin-6 expression after repeated bouts of eccentric exercise. International journal of sports medicine, v. 24, n. 01, p. 15-21, 2003.

XAVIER, Hermes T. et al. V Diretriz brasileira de dislipidemias e prevenção da aterosclerose. Arquivos brasileiros de cardiologia, v. 101, n. 4, p. 1-20, 2013.

XIA, Qianghua; GRANT, Struan FA. The genetics of human obesity. Annals of the New York Academy of Sciences, v. 1281, n. 1, p. 178-190, 2013.

ZEE, R. Y.L. et al. Association of a polymorphism of the angiotensin I-converting enzyme gene with essential hypertension. Biochemical and biophysical research communications, v. 184, n. 1, p. 9-15, 1992. 
ZHANG, C. et al. Common variants of the endothelial nitric oxide synthase gene and the risk of coronary heart disease among US diabetic men. Diabetes, v. 55, n. 7, p. 2140-2147, 2006.

ZHEN, J. et al. Association of ApoE Genetic Polymorphism and Type 2 Diabetes with Cognition in Non-Demented Aging Chinese Adults: A Community Based Cross-Sectional Study. Aging and disease, v. 9, n. 3, p. 346, 2018. 